OAI-PMH: http://www.indteca.com/ojs/index.php/Revista Scientific/oai

Artículo Original / Original Article

\title{
Adaptación social al proceso de retorno a la educación particular presencial
}

\author{
Autores: César Raúl Méndez Carpio \\ Universidad Católica de Cuenca, UCACUE \\ cmendezc@ucacue.edu.ec \\ Cuenca, Ecuador \\ https://orcid.org/0000-0003-0582-0107 \\ Natalia Adriana Palacios Montero \\ Universidad Técnica Particular de Loja, UTPL \\ npalacios@napalogistic.com \\ Cuenca, Ecuador \\ https://orcid.org/0000-0001-6625-2559
}

\section{Resumen}

El objetivo de la presente investigación, pretende indagar sobre la dinámica de las familias de estudiantes de nivel medio, la situación académica actual en casa y su eventual retorno a la educación con la modalidad presencial y uso de instalaciones en instituciones con financiamiento particular o fiscomisional. La investigación adoptó el enfoque cualitativo de carácter descriptivo en el que participaron una muestra de 971 padres de familia y/o representantes estudiantiles con características similares de educación particular en la zona urbana de Cuenca, Ecuador. Luego de obtener los resultados producto de instrumentos cualitativos como encuestas estructuradas y análisis de información documental, se obtuvieron datos que permitieron describir criterios de adaptación social a los procesos académicos de los estudiantes en sus hogares durante la actual pandemia, la realidad de la salud familiar y el posible contacto con personas contagiadas o con vulnerabilidad de contagio en el sector de influencia. Así mismo el estudio, facilitó el planteamiento de algunas consecuencias respecto al retorno progresivo o futuro a las aulas físicas, resaltando los criterios expresados por la muestra estudiada que servirán de preámbulo para la toma de decisiones por parte de las autoridades educativas zonales y distritales en este campo académico para el nivel medio.

Palabras clave: adaptación social; retorno progresivo; pandemia.

Cómo citar este artículo:

Méndez, C., \& Palacios, N. (2021). Adaptación social al proceso de retorno a la educación particular presencial. Revista Scientific, 6(19), 64-81, e-ISSN: 2542-2987. Recuperado de: https://doi.org/10.29394/Scientific.issn.2542-2987.2021.6.19.3.64-81

Fecha de Recepción: 02-09-2020
Fecha de Aceptación: 28-12-2020
Fecha de Publicación: 05-02-2021 
OAI-PMH: http://www.indteca.com/ojs/index.php/Revista Scientific/oai

Artículo Original / Original Article

\title{
Social adaptation to the return process to face-to-face private education
}

\begin{abstract}
The objective of this research aims to investigate the dynamics of the families of middle-level students, the current academic situation at home and their eventual return to education with the face-to-face modality and use of facilities in institutions with private or fiscal-commission financing. The research adopted a qualitative, descriptive approach in which a sample of 971 parents and / or student representatives with similar characteristics of private education in the urban area of Cuenca, Ecuador participated. After obtaining the results of qualitative instruments such as structured surveys and analysis of documentary information, data were obtained that allowed describing criteria of social adaptation to the academic processes of students in their homes during the current pandemic, the reality of family health and possible contact with people infected or vulnerable to contagion in the sector of influence. Likewise, the study facilitated the approach of some consequences regarding the progressive or future return to physical classrooms, highlighting the criteria expressed by the studied sample that will serve as a preamble for decisionmaking by the zonal and district educational authorities in this academic field for the intermediate level.
\end{abstract}

Keywords: social adaptation; progressive return; pandemic.

\footnotetext{
How to cite this article:

Méndez, C., \& Palacios, N. (2021). Social adaptation to the return process to face-toface private education. Revista Scientific, 6(19), 64-81, e-ISSN: 2542-2987. Recovered from: https://doi.org/10.29394/Scientific.issn.2542-2987.2021.6.19.3.64-81
}

Date Received: 02-09-2020
Date Acceptance: 28-12-2020
Date Publication: 05-02-2021 


\section{Introducción}

Sabemos que la situación mundial que estamos atravesando por la pandemia, ha tenido un gran impacto para todas las personas de la sociedad $y$ ello ha requerido adaptación y flexibilidad conforme avanzamos $y$ procuramos un retorno a nuestras actividades normales. Frente a ello, el Ministerio de Educación y Cultura del Ecuador considerando que ha existido y se mantiene en los sectores fiscales altos niveles de deserción escolar producto de varios factores, ha dispuesto que se elaboren planes institucionales de continuidad educativa, de retorno y uso progresivo a las instalaciones educativas previos diagnósticos situacionales y de ubicación de estudiantes que se han retirado del proceso educativo en las distintas instituciones del país considerando variables de bioseguridad a plantearse para recuperar los niveles de alfabetización conseguidos en los últimos años.

La primera variable del estudio intenta discernir la situación de adaptación social actual frente al cambio radical de la modalidad de estudios obligado por la pandemia en las familias de la urbe cuencana y analiza teorías y constructos que permitirán solventar las inquietudes acerca de la educación tradicional su incidencia a través del tiempo y la modalidad virtual como proceso de innovación tratado desde hace algunos años atrás y que hoy son el referente de la Pedagogía y didáctica aplicada en todos los niveles académicos de los planteles educativos en la ciudad, país, región y en el mundo.

Frente a esta situación provocada por la emergencia sanitaria ocasionada por el COVID-19 la cual es una patología infecciosa, declarada como pandemia por la OMS en marzo del año 2020 y, como consecuencia del crecimiento de dicha patología en todos los campos sociales y principalmente a la educación, Petrelli, Isacovich y Mattioni (2020a): afirman que, la educación se vio forzada en el mundo, a aplicar cambios drásticos en los métodos de enseñanza migrando de lo tradicional a lo virtual a pasos agigantados. 
Sin embargo, esta adecuación ha significado un gran desafío tanto para maestros como para alumnos, como lo conciben Carrasco y Baldivieso (2016a): al aplicarse los procesos educativos con el uso tecnológico se ha dado un cambio sustancial en la pedagogía moderna y ello afecta a sectores vulnerables que no tienen acceso a equipos ni herramientas electrónicas necesarias para su inclusión lo que ha obligado a las instituciones educativas a tomar acciones en beneficio de dichos sectores.

Los procesos de adaptación social en este sentido entonces forman parte del día a día de las actividades educativas en todos los niveles. Es necesario notar lo que afirma Moreno-Correa (2020a): quien concuerda que "el uso de aulas virtuales para mantener la comunicación con los estudiantes, plantear ejercicios, actividades, trabajos y guías para el trabajo independiente, es una estrategia acertada que asegura el acompañamiento y el aprendizaje" (pág. 20); frente a ello la humanidad se está adaptando al paso a la modalidad virtual, provocando un vuelco completo al concepto del modelo tradicional.

No hasta hace mucho, se hablaba de procesos de innovación educativa que comúnmente se vinculaba al tópico de la educación virtual, tema que Yong, Nagles, Mejía y Chaparro (2017a): conduce su estudio y afirma que el avance tecnológico inmerso en el campo académico tiene como base el uso del ciberespacio en un medio en donde se adquieren los conocimientos aplicando metodologías que aprovechan la utilización de las Tecnologías de Información y Comunicación basadas en el paso del entorno físico al virtual. Por otro lado, Moreno-Correa (2020b), está de acuerdo con que:

Además de ser eficaz, la educación virtual debe ser eficiente, factible y asequible, para esto se debe estructurar el curso, los objetivos de aprendizaje, la adaptación de las tecnologías de la información y la comunicación (TIC) y su uso pedagógico y tecnológico para que cada herramienta que se utilice se adapte a dichos objetivos (pág. 20).

Reforzando esta idea, Agüero (2019): reafirma que "la didáctica en la 
actualidad es quien se encarga de organizar las reglas de la metodología a utilizar para que el aprendizaje fluya eficientemente" (pág. 159). Por lo tanto, el proceso de enseñanza aprendizaje no depende en gran medida del espacio en el que se desarrolle sino de la metodología aplicada por los docentes enlazados a una didáctica aplicada de forma eficiente.

El paso entre la modalidad presencial y la virtual por lo tanto depende de las capacidades y características que tengan injerencia en el hecho educativo tales como: los métodos, las técnicas, los recursos y diría además los talentos que, juntos podríamos tratarlos como una pedagogía virtual y su aplicación procesual. Un claro ejemplo de una pedagogía virtual se evidencia en la aplicación del aula invertida que nuevamente Moreno-Correa (2020c): aporta señalando como una estrategia didáctica que invita al estudiante al desarrollo del pensamiento crítico con lo cual le permitirá llegar a resolver problemas cotidianos. En relación con la metodología de aula invertida, Carrasco y Baldivieso (2016b): dicen en cambio que: el estudiante debe llegar a sus clases con el apoyo previo de lecturas, análisis de videos, organizadores gráficos, entre otros; con el propósito de estar acorde a un aprendizaje activo en relación con su rol protagónico.

Con ello es necesario indicar que la educación virtual es producto del paso de la educación tradicional a distancia, que como conceptualizan Yong, Nagles, Mejía y Chaparro (2017b): la educación virtual es un punto en que se conectan la educación audiovisual tradicional o epistolar la cual utiliza recursos impresos y, la llamada educación interactiva que basa su estudio en la web y sus aplicaciones tales como el aprovechamiento de blogs, wikis, redes sociales, páginas colaborativas que llegan incluso a consumir recursos de la web 3.0 como integración de mundos virtuales, telepresencia, inteligencia artificial, etc.

De acuerdo con Carrasco y Baldivieso (2016c): al respecto comparan que: en la modalidad de enseñanza presencial, prima la comunicación 
sincrónica basada en acuerdos de fechas y horarios para que se presenten los procesos tanto de aplicación como evaluativos; en cambio en los ambientes virtuales se enfrentan a los momentos mayormente asincrónicos y el aprendizaje depende de la autonomía de los tiempos de aprendizaje de acuerdo a los intereses del estudiante quien es dueño de su propio aprendizaje lo que además puede afectar la adaptación a este sistema de aprendizaje y en ocasiones provocar frustración.

El estudio de Yong, Nagles, Mejía y Chaparro (2017c): sobre el avance progresivo de la educación virtual, menciona además que: es preciso considerar los cambios que la educación presenta actualmente siendo enfáticos en contar con un personal suficientemente capacitado en la metodología e-learning (aprendizaje electrónico), la tutoría virtual y todos los procedimientos que ello representan como son la preparación de herramientas virtuales, planificación y ejecución del hecho educativo en plataformas, actividades de gestión administrativa en entornos tecnológicos, entre otros propios de la labor académica. Lo que se menciona conlleva a que la educación virtual debe ser planificada con todos sus componentes diferenciadores para que los efectos y logros sean los necesarios para conseguir un óptimo desarrollo del aprendizaje virtual.

En el contexto del COVID-19, Petrelli, Isacovich y Mattioni (2020b): analizan que: se ha afectado en gran medida la educación tradicional producto del confinamiento social tanto preventivo como el obligado por los organismos de control. Sin embargo, en los contextos académicos de varios países de Latinoamérica y gracias al desarrollo tecnológico que se ha venido implementando en este campo; numerosas instituciones educativas pudieron adaptarse y dispusieron a sus integrantes, la continuidad pedagógica mediante la adecuación de la programación académica a entornos virtuales.

En el estudio de Flores y Garrido (2019): respecto al uso de las tecnologías en el campo académico, recalcan que "las Tecnologías de la 
Información y la Comunicación (TIC) son catalizadores que transforman y modifican los actuales escenarios de aprendizaje" (pág. 46); por lo que su aplicación eficiente permitirá conseguir una educación de calidad.

No obstante, con la crisis mundial de la pandemia que se está viviendo, varios estudiantes presentan dificultades en sus procesos de formación al no poder acceder a las aulas virtuales y otras herramientas al no disponer de equipos electrónicos personales llegando a formar parte del sector vulnerable en la llamada innovación tecnológica. Este aspecto es notado como un factor de incidencia letal en sectores rurales y la consecuencia en muchos casos, ha sido la falta de continuidad en el servicio educativo llegando a considerarse y a aplicarse, el cierre de centros educativos por la alta deserción estudiantil

Al respecto, el avance progresivo en el cierre de muchas instituciones educativas especialmente de tipo particular, están provocando una discontinuidad educativa en varios millones de estudiantes de varios países en el mundo, e inclusive ha influido en el despido de docentes inmersos en este sector educativo. A pesar de ello, los líderes de gobiernos democráticos han adoptado medidas que permitan mitigar esta situación regularizando sus economías en beneficio de la educación y tratando de brindar servicios de internet o ayudas económicas con el propósito de palear el problema y facilitar el acceso a una parte de la población para que accedan de manera sencilla a la educación virtual.

La realidad es diferente para los estudiantes de los centros educativos particulares y por qué no citar algunos fiscomisionales, es decir, de economía compartida entre el gobierno y lo privado que, han sorteado las consecuencias de la pandemia y han podido adaptarse tanto con su contingente humano o su personal como con los recursos tecnológicos propiedad de las instituciones y de los estudiantes a través de sus padres de familia y/o representantes.

Las instituciones de control del sistema educativo del Ecuador, preocupadas por la situación de las familias y las consecuencias fatales de 
deserción estudiantil que están dejando los efectos de la falta de recursos y accesos al sistema educativo virtual, especialmente en los sectores carentes de los recursos tecnológicos necesarios como es el sector rural fiscal y sorteando múltiples variables, han visto la necesidad de tomar acciones y medidas emergentes con el fin de mitigar el impacto y conseguir a través de planes institucionales de continuidad educativa que resulten por fases definidas, el retorno progresivo a ocupar las instalaciones físicas tratando de recuperar la población desertora.

Las fases de reintegración tienen que ver con un primer momento en que los directivos y docentes ocupen los predios con las medidas de seguridad necesaria, los cuáles planificaran y prepararan los espacios y recursos que de manera progresiva servirán de base para la reinvención creciente de sus estudiantes, sin dejar de lado aquellos que por situaciones de vulnerabilidad no pueden retornar y hay que considerar las estrategias para mantenerles estudiando en la modalidad virtual.

Esta variable de análisis ha permitido completar el desarrollo del presente estudio y dar respuesta a la pregunta de investigación de si ¿están listos los padres de familia y/o representantes de los estudiantes de las unidades educativas del sector urbano particular en que sus representados retornen progresivamente a la educación presencial?

En síntesis, el objetivo que da curso a la investigación trata de determinar el afrontamiento social en función de la adecuación a los procesos educativos actuales producto del confinamiento de las familias, frente a la decisión de retomar la modalidad presencial mediante el uso progresivo de las instalaciones físicas de las instituciones educativas, todo ello con el propósito de informar el gran impacto que ha tenido el cambio en la gestión de las instituciones educativas al poder adecuarse a la modalidad de educación virtual provocado por la pandemia del COVID-19.

El cambio dado afecta considerablemente a toda la comunidad 
educativa, el personal docente además ha tenido que someterse a procesos de capacitación enfocados en la pedagogía virtual y el uso de herramientas web en beneficio de los estudiantes de los distintos niveles de estudio aprendiendo el uso de software en función de la edad cronológica de sus alumnos y el desarrollo motriz, así también mantener información permanente por los distintos medios de comunicación en labores compartidas con sus pares académicos y padres de familia representantes de dichos estudiantes quienes también deben adecuar sus tiempos y modelos de seguimiento al desarrollo académico en sus familias. Todo lo analizado indica que los miembros de la comunidad educativa se han adaptado al cambio y se nota en las familias investigadas, una reacción contraria al retorno inmediato a las aulas o instalaciones físicas de las Instituciones Educativas.

\section{Metodología (Materiales y métodos)}

Se consideró para el proceso metodológico de esta investigación al enfoque cualitativo de tipo descriptivo ya que se apoyó en técnicas de recolección de la información a través de encuestas estructuradas a un número determinado de familias de la Ciudad de Cuenca, Ecuador y que tienen a sus hijos y/o representados estudiando el nivel inicial, de educación general básica y bachillerato en instituciones de tipo particular o fiscomisional. Así mismo se optó por la técnica de revisión bibliográfica, observando varias fuentes documentales en bases de datos digitales sobre los temas producto de las variables investigadas.

Se planteó una muestra probabilística en el Sector educativo de la ciudad de Cuenca al cuál, se pudo aplicar una encuesta de modalidad online permitiendo captar las inquietudes y pensamientos de las familias cuencanas frente a la situación académica actual y sus reacciones ante un eventual retorno al uso de las instalaciones físicas dispuesto por el Ministerio de Educación del Ecuador planteado en el llamado plan institucional de 
continuidad educativa permanencia escolar y uso progresivo de las instalaciones de las instituciones educativas conociendo la situación fenomenográfica presente.

Se notó una gran cantidad de información publicada en las bases de datos digitales referentes a los procesos y características de la situación y realidad actual frente a la pandemia producida por el COVID-19 y que ha dado giros completos a la didáctica educativa en todos los niveles académicos de la sociedad cuencana. Todo el proceso metodológico aplicado permitió desarrollar el objetivo de documentar los criterios acerca del posible retorno a las actividades escolares presenciales en las instituciones educativas en la ciudad de Cuenca.

\section{Resultados (análisis e interpretación de los resultados)}

El paso del tiempo, la conformidad de la adaptación a los procesos tradicionales educativos, el avance tecnológico y la anterior reacción lenta a aceptar la educación virtual en los niveles actuales por diferentes motivos como la falta de recursos, fueron causas para que la integración de la modalidad virtual y sus bondades hayan sido aceptadas a la fuerza hoy en día producto de la pandemia mundial en las familias que mantienen a parte de sus integrantes estudiando en los distintos centros educativos de la ciudad y el país.

La aplicación de la encuesta estructurada permitió manejar variables de tipo situacional como: cursos por niveles y paralelos en los que se ubican a los estudiantes dentro de las unidades educativas siendo equitativa la muestra en los distintos años de formación, se recabaron además los correos electrónicos personales que aportan a la retroalimentación de los datos. En relación con las preguntas objetivas para el análisis del objeto de estudio, se pueden observar de manera porcentual los resultados en la tabla 1. 
Instituto Internacional de Investigación y Desarrollo Tecnológico Educativo INDTEC, C.A.

DOI: https://doi.org/10.29394/Scientific.issn.2542-2987.2021.6.19.3.64-81

OAI-PMH: http://www.indteca.com/ojs/index.php/Revista Scientific/oai

Artículo Original / Original Article

Tabla 1. Resultados de la encuesta para analizar retorno a clases presenciales.

\begin{tabular}{|c|c|c|c|}
\hline CUESTIONARIO PREGUNTAS OBJETIVAS & PARCIAL & SI & NO \\
\hline $\begin{array}{l}\text { 1. ¿Está de acuerdo Ud. en que se inicie un proceso de retorno } \\
\text { progresivo a las actividades educativas presenciales y uso de } \\
\text { instalaciones en las próximas fechas? }\end{array}$ & & $31,8 \%$ & $68,2 \%$ \\
\hline \multicolumn{4}{|c|}{$\begin{array}{l}\text { 2. En caso de que su respuesta anterior sea SI, señale la actividad que considere más } \\
\text { adecuada: }\end{array}$} \\
\hline a. No asistir. & $67,6 \%$ & & \\
\hline b. Asistir toda la jornada. & $7,9 \%$ & & \\
\hline c. Asistir toda la jornada dos veces por semana. & $16,5 \%$ & & \\
\hline d. Asistir media jornada todos los días. & $8,1 \%$ & & \\
\hline \multicolumn{4}{|l|}{ 3. Considera adecuado que el plan de retorno progresivo inicie en: } \\
\hline a. Cuando exista una vacuna. & $52,4 \%$ & & \\
\hline b. Siguiente Quimestre. & $16,9 \%$ & & \\
\hline c. Siguiente año lectivo & $12,9 \%$ & & \\
\hline $\begin{array}{l}\text { d. Cuando las autoridades dispongan (COE cantonal y } \\
\text { nacional). }\end{array}$ & $12,6 \%$ & & \\
\hline e. Lo antes posible. & $\%$ & & \\
\hline $\begin{array}{l}\text { 4. ¿Está de acuerdo con que las actividades académicas del } \\
\text { plantel se mantengan en la Fase } 1 \text { es decir el estudio desde } \\
\text { casa con las plataformas tecnológicas con las que cuenta la } \\
\text { Unidad Educativa? }\end{array}$ & & $90,8 \%$ & $9,2 \%$ \\
\hline $\begin{array}{l}\text { 5. ¿Considera que se puede adecuar con sus equipos } \\
\text { electrónicos a la continuidad de las clases de manera online o } \\
\text { virtual? }\end{array}$ & & $92,2 \%$ & $7,8 \%$ \\
\hline $\begin{array}{l}\text { 6. ¿Existen dentro de su familia personas vulnerables frente a } \\
\text { la pandemia? (adultos mayores, personas con enfermedades } \\
\text { catastróficas, enfermedades comprometidas como diabetes, } \\
\text { hipertensión arterial, otras). }\end{array}$ & & $68 \%$ & $32 \%$ \\
\hline $\begin{array}{l}\text { 7. ¿Ha pasado o está pasando por el proceso de contagio del } \\
\text { COVID-19? }\end{array}$ & & $26,8 \%$ & $73 \%$ \\
\hline a. estudiantes. & $1,2 \%$ & & \\
\hline b. padres de familia. & $8,4 \%$ & & \\
\hline c. miembros del grupo familiar. & $17,2 \%$ & & \\
\hline d. un conocido. & $73,2 \%$ & & \\
\hline $\begin{array}{l}\text { 8. ¿Ha tenido contacto en los últimos días con personas que } \\
\text { se enteró que han dado positivo a la prueba del COVID-19? }\end{array}$ & & $22,5 \%$ & $77,5 \%$ \\
\hline $\begin{array}{l}\text { 9. ¿Está dispuesto a enviar a los cadetes con los medios de } \\
\text { bioseguridad requeridos tales como: mascarillas KN95, alcohol } \\
\% \text { adecuado, gel desinfectante, etc.? }\end{array}$ & & $61,6 \%$ & $38,4 \%$ \\
\hline $\begin{array}{l}\text { 10. ¿Está de acuerdo en que la Institución educativa devuelva } \\
\text { a su domicilio a los estudiantes que no cuenten con los medios } \\
\text { de biosequridad necesarios indicados anteriormente? }\end{array}$ & & $88 \%$ & $12 \%$ \\
\hline
\end{tabular}

Fuente: Los Autores (2020).

Con la presente investigación, se identificaron diferentes hallazgos fruto 
de las 971 familias que respondieron la encuesta y los datos expuestos indican que un total de 659 familias que equivalen al 68,2\% de la población consultada señalan no estar de acuerdo en que se inicie un proceso de retorno progresivo a las actividades educativas presenciales y uso de instalaciones en las próximas fechas; el 31,8\% restante afirman estar de acuerdo con ello.

Se nota también que concomitante a lo señalado anteriormente, el $67,6 \%$ indican que lo mejor es no asistir a la modalidad presencial mientras que el porcentaje restante divide su criterio de la siguiente manera: el 7,9\% considera que se debe asistir toda la jornada, el 16,5\% dice que dos veces por semana, el 8,1\% que debe asistir media jornada todos los días de la semana. En caso de que se aplique un plan de retorno progresivo, están de acuerdo en enviar a los estudiantes a la institución educativa en cuanto exista una vacuna 506 familias que corresponden al 52,4\% mientras que el 16,9\% están dispuestos a enviarlos en el siguiente quimestre académico, el 12,9\% en el siguiente año lectivo, el $12,6 \%$ en cuanto las autoridades nacionales lo dispongan y un porcentaje menor correspondiente al 5,2\% afirman que lo antes posible.

Con relación a que si están de acuerdo con que las actividades académicas del plantel se mantengan con el estudio desde casa con el uso de las plataformas tecnológicas con las que cuenta la Unidad Educativa, se manifiestan el 90,8\% y apenas el 9,2\% dice estar en desacuerdo y en relación con ello se nota que la gran mayoría de familias con sus estudiantes se han adecuado a la continuidad de sus clases virtuales con los equipos tecnológicos que disponen en sus domicilios afirmándolo el $92,2 \%$, pensando lo contrario el $7,8 \%$ de la población restante.

Se pudo además identificar en el estudio que un total de 657 representantes de los estudiantes; el 68\%, tienen en su entorno familiar personas vulnerables frente a la pandemia: adultos mayores, personas con enfermedades catastróficas, enfermedades comprometidas como diabetes, 
hipertensión arterial entre otras, y el 32\% complementario no dicen sufrir esta situación. De lo que se contempla entonces en el núcleo familiar, han tenido situaciones de contagio el $26,8 \%$ y el $73 \%$ no presentan signos adversos, desagregado en el $1,2 \%$ de estudiantes que se han contagiado, el $8,4 \%$ de padres de familia y el 17,2\% como miembro del grupo familiar y además se dice que el $73,2 \%$ conoce a personas del barrio o de distintos sectores, que están pasando por la situación del contagio del virus COVID-19.

Si se anota además que, con las medidas de seguridad tomadas en los diferentes entornos de la ciudad, han tenido contacto con personas que han dado positivo a la prueba del COVID-19 un $22,5 \%$ a diferencia del $77,5 \%$ que afirman no conocer el dato. Por otro lado, al ver la disponibilidad de aseguramiento de la seguridad de los estudiantes, el 61,6\% de padres de familia y/o representantes estudiantiles afirman que en caso de aplicarse un plan de retorno al uso de instalaciones, están dispuestos a enviar a sus representados con los medios de bioseguridad requeridos tales como mascarillas KN95, alcohol \% adecuado, gel desinfectante, etc., y el $38,4 \%$ dice no estar de acuerdo lo que genera preocupación por la falta de cuidado demostrado aunque también afirma el $88 \%$ estar de acuerdo en que la Institución educativa devuelva a su domicilio a los estudiantes que no cuenten con los medios de bioseguridad necesarios indicados anteriormente siendo el $12 \%$ restante de encuestados los mantener medidas no seguras para sus hijos y/o estudiantes representados.

Por lo resultante de la información recabada, se nota que las familias cuencanas que tienen estudiando a sus hijos y/o representados en las instituciones educativas de la urbe, han desarrollado normas de adaptación social a la educación en línea con los recursos con los que disponen y se vuelven reacios a la ocupación inmediata o mediata de las instalaciones físicas creadas para el hecho académico. 


\section{Conclusiones}

Por los resultados expuestos en el análisis de datos de la información recabada a través de los instrumentos, se nota un gran nerviosismo y falta de confianza en los protocolos de retorno a las actividades académicas presenciales por parte de padres de familia y/o representantes de las instituciones educativas particulares de nivel medio de la zona urbana de Cuenca, Ecuador.

Se notó que la mayoría de las familias se han adaptado a los procesos de aprendizaje virtual de una manera u otra y que el cambio radical dado en el mundo a la educación no presencial ha permitido acostumbrarse a esta realidad y causa reacción al tratar de volver al menos en días cercanos a nuevas formas de uso de las instalaciones físicas en las condiciones que se planifiquen por parte de autoridades locales o de gobierno. Por tanto, la adaptación social a los procesos académicos como se la llama en los casos investigados podríamos afirmar ha sido un éxito, lo que frena sustancialmente la utilización progresiva y el retorno a la antigua realidad académica con modalidad presencial o semipresencial.

Basados en los datos emitidos a través de las consultas realizadas, se puede evidenciar que la realidad de la salud familiar en la ciudad de Cuenca presenta altos porcentajes de riesgo en cuanto a la vulnerabilidad de sus miembros como en tanto al contacto con personas contagiadas del virus que produce el nombrado COVID-19, resultando los criterios de que mientras no exista una vacuna no están dispuestos a retornar a las labores académicas presenciales ofertadas por las instituciones educativas particulares de la urbe cuencana.

Se menciona además consecuencias a corto y mediano plazo que podrían ser fatales en el rebrote de altos niveles de contagio, considerando que no están adecuadas en su totalidad las garantías de bioseguridad puesto que muchos padres de familia y/o representantes no están de acuerdo o no 
tienen acceso a los mejores recursos de protección como por ejemplo mascarillas diarias tipo KN95, alcohol y gel antiséptico de porcentajes adecuados, protectores faciales, trajes externos anti fluido, entre otros necesarios para actuar ante un contacto masivo de personas dentro de las instalaciones.

Con estas condiciones y en consecuencia de lo investigado, no se recomienda en el corto plazo el retorno y uso de instalaciones educativas al menos en el sector de influencia analizado como son los centros educativos particulares de Cuenca que disponen de garantías tecnológicas frente a la continuidad educativa de sus estudiantes los cuales han demostrado haberse adaptado a los nuevos requisitos con el uso diario de sus equipos electrónicos personales y familiares notados en sus menciones.

\section{Referencias}

Agüero, M. (2019). La Transcomplejidad como Agente Integrador de las Tecnologías de Información y Comunicación. Revista Scientific, 4(11), 156-176, e-ISSN: 2542-2987. Recuperado de:

https://doi.org/10.29394/Scientific.issn.2542-2987.2019.4.11.8.156-176

Carrasco, S., \& Baldivieso, S. (2016a,b,c). Educación a distancia sin distancias. Universidades, (70), 7-26, e-ISSN: 0041-8935. Recuperado de: https://www.redalyc.org/articulo.oa?id=37348529003

Flores, D., \& Garrido, J. (2019). Competencias digitales para los nuevos escenarios de aprendizaje en el contexto universitario. Revista Scientific, 4(14), 44-61, e-ISSN: 2542-2987. Recuperado de: https://doi.org/10.29394/Scientific.issn.2542-2987.2019.4.14.2.44-61

Moreno-Correa, S. (2020a,b,c). En respuesta a la carta el editor «A propósito del artículo "La innovación educativa en los tiempos del Coronavirus"». Salutem Scientia Spiritus, 6(1). 19-21, e-ISSN: 24631426. Recuperado de: 
https://revistas.javerianacali.edu.co/index.php/salutemscientiaspiritus/a rticle/view/2390

Petrelli, L., Isacovich, P., \& Mattioni, M. (2020a,b). Estudiar y trabajar en la universidad en contextos de aislamiento social, preventivo y obligatorio. En Desigualdades en el Marco de la Pandemia: Reflexiones y Desafíos. Argentina: Universidad Nacional de José Clemente Paz. Recuperado de: https://www.unpaz.edu.ar/node/3932

Yong, É., Nagles, N., Mejía, C., \& Chaparro, C. (2017a,b,c). Evolución de la educación superior a distancia: desafíos y oportunidades para su gestión. Revista Virtual Universidad Católica del Norte, (50), 80-105, eISSN: 0124-5821. Recuperado de:

https://www.redalyc.org/articulo.oa?id=194250865006 


\section{César Raúl Méndez Carpio \\ e-mail: $\underline{\text { cmendezc@ucacue.edu.ec }}$}

Nacido en Cuenca, Ecuador, el 22 de febrero del año 1971.

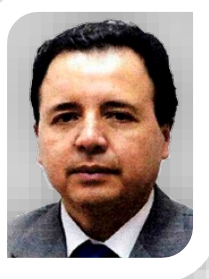

Dr. En Ciencias de la Educación, especialidad Ordenadores en la Universidad Católica de Cuenca (UCACUE); Magister en Docencia y Currículo para la Educación Superior en la Universidad Técnica de Ambato (UNITA); Magister en Administración de Empresas en la Universidad del Azuay (UDA); Actualmente me desempeño como Jefe de Investigación en el Colegio Militar "Abdón Calderón" y como docente en la Universidad Católica de Cuenca (UCACUE), en las Asignaturas: Estadística Aplicada, Metodología de Investigación, Tecnologías de Información y Comunicación. 


\section{Natalia Adriana Palacios Montero \\ e-mail: npalacios@napalogistic.com}

Nacida en Cuenca, Ecuador, el 21 de septiembre del año

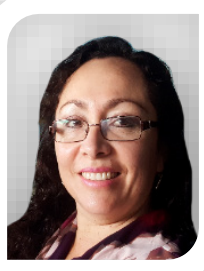

1977. Me gradué en el Colegio Manuela Garaicoa de Calderón, en la especialidad de Secretariado Español; soy Auditora de normas BASC para el capítulo Azuay; Actualmente me desempeño como General Manager en la Empresa NAPALOGISTIC IFF, que es un Freight Forwarders o Agente de Carga Internacional.

El contenido de este manuscrito se difunde bajo una Licencia de Creative Commons ReconocimientoNoComercial-Compartirlgual 4.0 Internacional 